\title{
A machine learning for environmental noise classification in smart cities
}

\author{
Yaseen Hadi Ali ${ }^{1}$, Rozeha A. Rashid ${ }^{2}$, Siti Zaleha Abdul Hamid ${ }^{2}$ \\ ${ }^{1}$ Department of Computer Techniques Engineering, Alsalam University College, Baghdad, Iraq \\ ${ }^{2}$ Telecommunication Software and Systems (TeSS) Research Group, Faculty of Engineering, Universiti Teknologi Malaysia, \\ Johor Bahru, Malaysia
}

\begin{tabular}{l} 
Article Info \\
\hline Article history: \\
Received Jul 17, 2021 \\
Revised Dec 26, 2021 \\
Accepted Jan 17, 2022 \\
\hline
\end{tabular}

Keywords:

Environmental noise

Machine learning

Noise classification

Smart cities

Urban noise

\begin{abstract}
The sound at the same decibel $(\mathrm{dB})$ level may be perceived either as annoying noise or as pleasant music. Therefore, it is necessary to go beyond the state-of-the-art approaches that measure only the $\mathrm{dB}$ level and also identify the type of the sound especially when the sound is recorded using a microphone. This paper presented a case study that considers the ability of machine learning models to identify sources of environmental noise in urban areas and compares the sound levels with the recommended levels by the World Health Organization (WHO). The approach was evaluated with a dataset of 44 sound samples grouped in four sound classes that are highway, railway, lawnmowers, and birds. We used mel-frequency cepstral coefficients for feature extraction and supervised algorithms that are Support vector machine (SVM), k-nearest neighbors $(\mathrm{KNN})$, bootstrap aggregation (Bagging), and random forest (RF) for noise classification. We evaluated performance of the four algorithms to determine the best one for the classification of sound samples in the data set under consideration. The findings showed that the noise classification accuracy is in the range of 95\%-100\%. Furthermore, all the captured data exceeded the recommended levels by WHO which can cause adverse health effects.
\end{abstract}

This is an open access article under the CC BY-SA license.

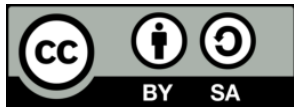

\section{Corresponding Author:}

Rozeha A. Rashid

Telecommunication Software and Systems (TeSS) Research Group, Faculty of Engineering

Universiti Teknologi Malaysia

Skudai 81310, Johor Bahru, Malaysia

Email: rozeha@utm.my

\section{INTRODUCTION}

With the increasing population of $60 \%$ by the end of 2030 [1], noise pollution will become a serious issue for many cities around the world. This is due to an increase in noise exposure (unpleasant, unwanted, and often loud) for the people who are living in cities. Continuous exposure to high levels of noise may cause psychological and physiological issues e.g. hearing impairment, high blood pressure, heart disease, inconvenience, and sleep annoyance [2]. Children who are living in noisy places showed low academic results [3]. The low birth weight of newborn babies is associated with noise exposure in pregnant women [3]. Therefore, there is an essential need not only to raise awareness of the harmful effects of noise pollution, but also to provide them with equipment to monitor their noise exposure. A professional sound level meter (SLM) is a standard tool for measuring sound pressure levels (SPL) in dB or dBA (A-weighted decibels to account for varying human ear's sensitivity to sound threshold). These tools are expensive, sensitive, bulky and above all, hard to use for non-professionals. However, in the era of industrial revolution 4.0, it seems promising to utilize modern technology such as a mobile application on a smartphone as the sensing tool for 
noise capture and preprocessing. With the development of smartphones, all of us carry a powerful minicomputer provided with different sensors, including microphones. These sensors can be used with a set of application programming interfaces (APIs) provided by the smartphone to create applications to measure sound pressure level [4], coronary heart disease detector [5], earth-quake detector [6], and pathological tremor detector [7]. However, it is not just necessary for noise measurement to be carried out, it is also essential to control the level of exposure to noise in critical areas such as schools, hospitals, and kindergartens. Hence, there is a need to have noise pollution measurement data to assess the level of noise exposure in certain areas.

Developing countries in asia like China, Vietnam, and India face major traffic noise pollution problems in their cities [8]. Malaysia is among the developing countries that are affected by this problem due to the rapid rate of growth and the increasing number of road transportation networks supporting the development process. Awareness of noise pollution is not a recent scenario as it has attracted the attention of authorities in Malaysia since 1979 [9]. According to the star online (2016), 132 complaints regarding noise pollution were reported to Malaysia's Department of Environment in 2015. The majority of complaints were about noise from commercial and construction sites. Other complaints were documented from industry and transportation systems.

According to the environmental control standards regulated by Malaysia's Department of Environment (2007) in residential and suburban areas, the maximum allowed noise level outside of lowdensity residential areas must not exceed 55 (dBA) to protect the community from this urban noise. However, the noise limits vary based on the sensitivity of the area, type of site, and the various rules adjust by the countries. Therefore, the assessment of specific noise levels will assist to understand the real situation of noise pollution in the residential area. Moreover, the obtained results contribute to the identification of adequate precautions to control noise disturbance in residential areas.

In Kuala Lumpur, which is the measurement site, the increasing population, and road transportation due to urban development and daily demand of the population has been directly related to urban noise pollution. Transportation-related noise pollution comes from a variety of sources, including traffic noise, railway noise, aircraft noise, and marine noise. Traffic noise is defined as any unpleasant sound produced by vehicles when it is running on roadways. It is also often recognized as the most prevalent and intense form of transportation of noise sources [10].

Since Kuala Lumpur is a tropical and cosmopolitan city that contributes to the Malaysian economy, it considers the noisiest state. The traffic noise mainly originates from the modified motorcycles and the effect of frictional between the road surface and vehicle tires which causes a strong negative effect to the urban communities and the surrounding environment [11], [12]. The predominance of traffic noise levels in the surrounding environment affects resting, sleeping, learning, and communication in the population, in addition to the disturbance of traffic noise, they create long-term threats to human health [13].

Machine learning is often cited as an effective means of analyzing complex datasets and it is also used to address the issue of noise pollution in smart cities. In this paper, we present an approach for noise classification in smart cities using machine learning. Four classifiers are used in this study that is support vector machine (SVM), k-nearest neighbors (KNN), bootstrap aggregation (Bagging), and random forest (RF). To evaluate the accuracy, we prepared a dataset of 44 samples of environmental sounds that are captured from the surrounding environment by noise capture mobile application in four different classes (including highway, railway, lawnmowers, and birds). This paper's major contributions include:

- A machine learning technique for noise classification.

- Evaluation of the approach using a recorded dataset of 44 samples of environmental sounds.

- Threshold test for the captured noise levels against WHO standards.

The rest of this paper is organized as follows. Section 2 gives an overview of machine learning and the environmental noise dataset. Section 3 describes the steps of the methodology used in this paper. Section 4 presents the findings of our approasch and the paper is concluded in section 5.

\section{THE PROPOSED PROCEDURE}

In this section, an overview of smart cities, machine learning, environmental noise dataset, and feature extraction are provided. Key fields and domains that constitute a smart city are presented. The decision to use supervised learning is explained. The sources of the environmental noise dataset are also given as well as the associated noise classification approach.

\subsection{Smart city}

According to the United Nations (2018), 55\% of the world's population lives in civilized areas and this is expected to increase to $68 \%$ by 2050 [14]. Rapid urbanization has raised several challenges and 
problems. The mission of smart cities is to mitigate such problems and optimize the use of resources. A "smart city" [15] is a developed urban area that uses information and communication with government and society collaboration, to respond to various urban challenges and thus enhance the quality of urban life. Common examples of smart city projects include automation, smart waste management services, the salubrity of public buildings, air quality monitoring, traffic congestion monitoring, noise monitoring, energy consumption monitoring, smart lighting, and smart parking. Despite the increased use of the term "smart city" in the last few years, there is still no absolute definition until now. According to the European smart cities project [16], smart cities can perform well in six major areas of urban development: smart government, smart economy, smart mobility, smart living, smart people, and smart environment. Figure 1 shows some domains from each key field.

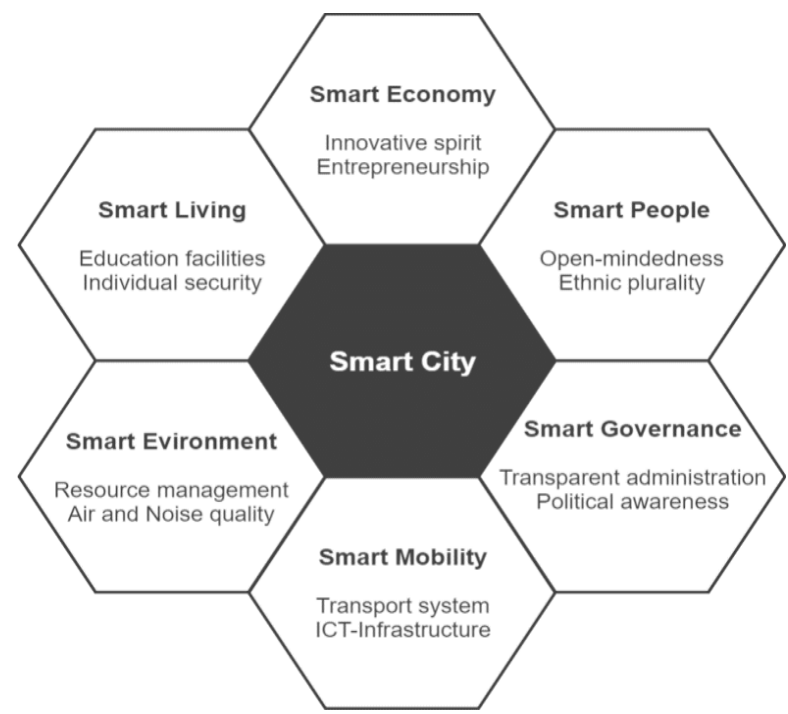

Figure 1. The key fields and some related domains of urban development needed for smart cities

\subsection{Machine learning}

Machine learning (ML) is a field of artificial intelligence (AI) that offers a system automatic learning and improvement ability from experience without programming it explicitly. It can be separated into two different categories: supervised learning and unsupervised learning. Supervised learning involves predicting an output for a given input by using a statistical model. In unsupervised learning, there is no supervised output. It is usually used to learn the structure of the data. The model developed in this study needs to classify the type of four different noise sources. Therefore, supervised learning is used. In the middle of the multiplicity of classification algorithms, choosing the right one is not straightforward since there is no ideal one that fits with all applications and there is always a trade-off between different model properties such as accuracy, complexity, memory usage, and training speed. This research focuses on the classification algorithms that are SVM, KNN, Bagging, and RF [17].

\subsection{Environmental noise dataset}

To study the performance of our approach, we conduct experiments with four different classes of environmental sounds; highway, railway, mechanical machines, and birds. This is because, from observation, noises from those sources are the prominent ones in the city of Kuala Lumpur. For this study, we capture noise from the surrounding environment by using the NoiseCapture mobile application [18] on a smartphone. This mobile application is adopted due to its flexibility, characteristics, and simplicity compared to other mobile applications. The total dataset contains 44 sound samples at various lengths. Table 1 provides the information about the environmental sound samples that we use for experimentation.

The data was collected for three days from different sources. The noise originated from highway and railway were recorded during morning, afternoon, and evening time. The noise data from birds were recorded during the evening time. The noise coming from mechanical machines (lawnmowers) was recorded during the morning and afternoon time. 
Table 1. Classes of sound samples in the dataset

\begin{tabular}{ccc}
\hline Class & Sample & Duration (Min.) \\
\hline Highway noise & 9 & 500 \\
Railway noise & 27 & 40 \\
Mechanical machine & 3 & 17 \\
Bird's noise & 5 & 90 \\
Total & 44 & $10 \mathrm{hrs} \mathrm{46} \mathrm{min}$ \\
\hline
\end{tabular}

\subsection{Feature extraction}

In this part, we describe our machine learning-based noise classification approach. The proposed noise classification system is illustrated in Figure 2. Mel frequency cepstral coefficients (MFCCs) are obtained from a training dataset of sound samples and used to train KNN, SVM, Bagging, and RF models which are used to predict the type of perceived ambient noise.

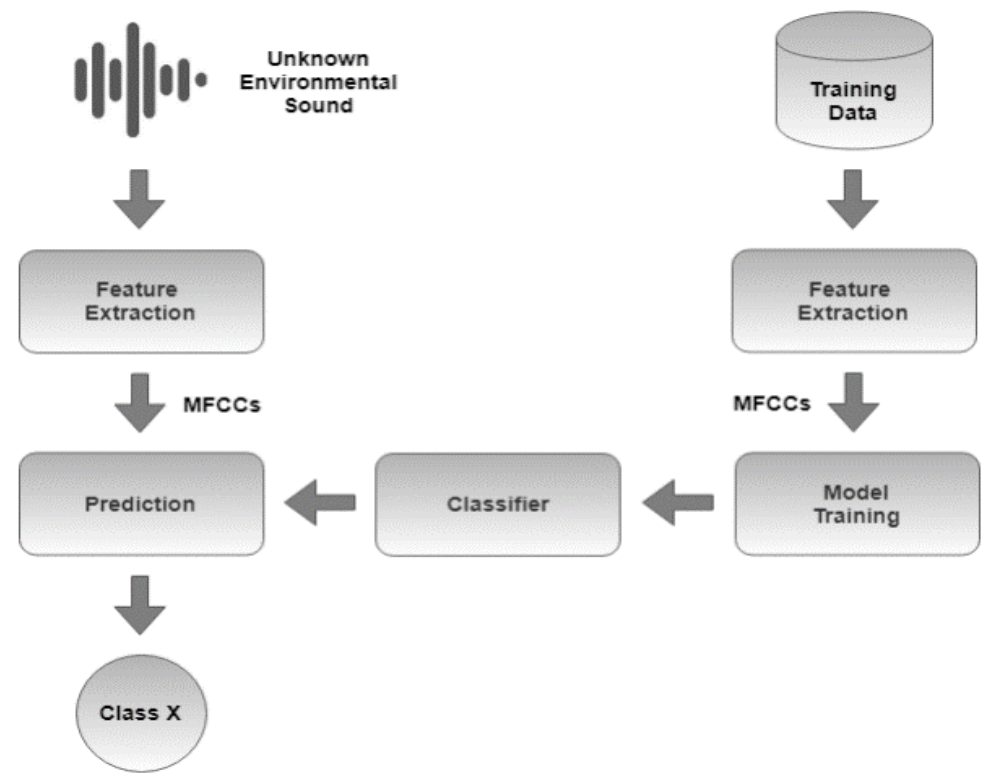

Figure 2. ML based approach for noise classification [19]

In an automatic noise classification system, the first step is feature extraction. MFCCs [20] is a wellknown feature set that is commonly employed in the field of sound classification due to their high correlation with what humans can hear. The approach illustrated in Figure 3 is used to obtain MFCCs.

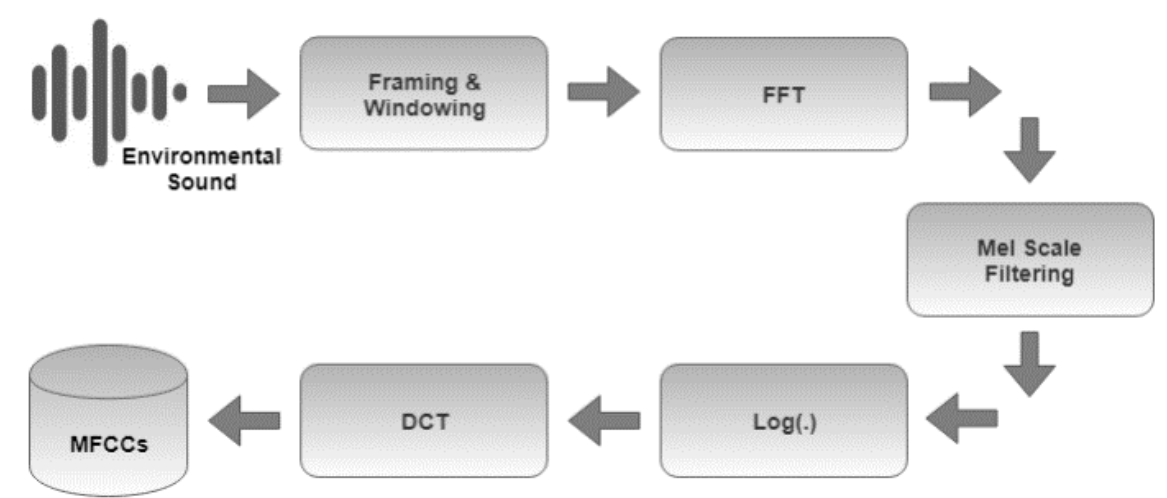

Figure 3. The technique for producing MFCCs of environmental sounds [19] 
In this research, we investigate the viability of performing feature extraction based on MFCCs. We calculate the first 12 MFCCs of all frames in the signal and add the frame energy to each feature vector. As a result, each audio stream is translated into a 13-dimensional feature vector sequence.

\section{METHOD}

\subsection{Training the classification model}

In this section, the model is constructed and trained using Scikit-learn, a library written in Python programming language. The library provides implementations for a range of machine learning algorithms [21]. The performance of four models that are SVM, KNN, Bagging, and RF is investigated on four different classes of environmental sounds: highway, railway, lawnmowers, and birds. Figure 4 depicts the flow chart of this procedure. The data-set of 44 samples of environmental sounds is divided into two sub-sets randomly: $67 \%$ is used for training and $33 \%$ for testing. The training data is used for developing and training the model while testing data is used for validating the developed model.

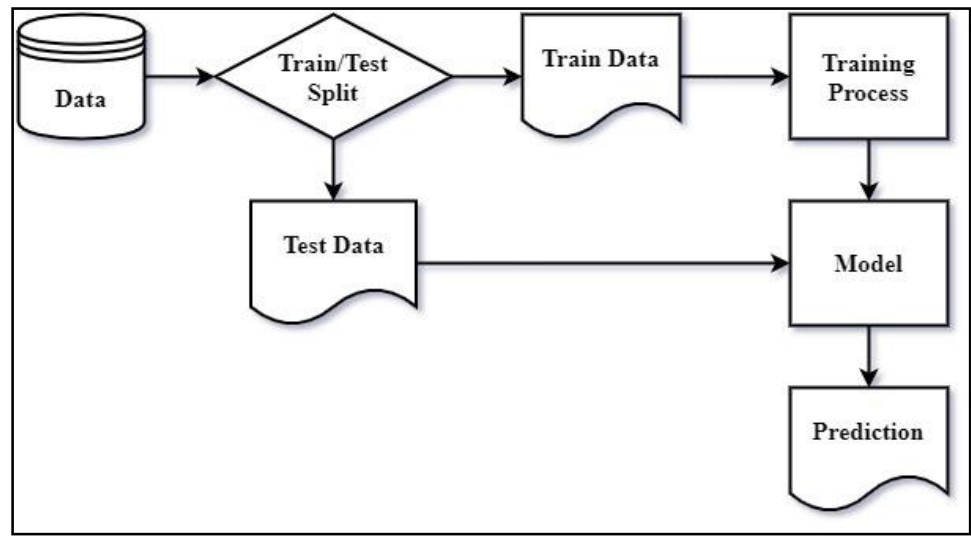

Figure 4. Flowchart of ML model development

\subsection{Machine learning life cycle}

Machine learning has given computer systems the capacity to learn automatically without being programmed directly. The ML method can be represented using the life cycle, which is a cyclical mechanism for creating a successful machine learning project. Knowing the problem is important before the beginning of the life cycle since a successful outcome depends on a better understanding of the problem. In the full life cycle process, an ML model is generated by having "training" to solve a problem. However, data is required to train a model, so the life cycle begins by gathering data.

Data gathering is one of the life cycle's most critical phases. The quantity and consistency of the data obtained will determine the production efficiency. The more data, the more reliable the forecast would be. The purpose of this phase is to identify multiple sources of data and to collect all data-related issues. In this study, the NoiseCapture mobile application will be installed on a smartphone (Huawei honor 6x) to capture noise data from the surrounding environment. It will be used to record four kinds of environmental noise originating from the highway, railway, mechanical machines (lawnmowers), and birds.

After data collection, data preparation is the next step where the data is explored and studied. To explain the essence, features, format, and consistency of data, data exploration is used. A greater interpretation of data adds to an efficient outcome. The smartphone picks up audio from its microphone and converts it to an audio buffer in a digital format composed of floating-point numbers equal to the sound pressure input. To use a smartphone as a sound level meter, the sound intensity level and frequency range must be measured from the audio input buffer from an application running on the smartphone.

The data is taken as a ZIP file containing 3 files [22]: a 'README.html' file detailing the metafiles of the two data files 'meta. properties' and 'track. geojson'. The first data file is in text format, containing general smartphone and device information, as well as the values of the LAeq (A-weighted equivalent continuous noise level over the recording time). The second file is in '.geojson' format (a common JSON encoded geospatial data interchange format and dedicated to vector data), containing measurement-related information for each of the measurement points (each point is specified in the world geodetic system 1984 (WGS 84) format by its latitude and longitude coordinates) [18]. 
To make it more suitable for analysis, data pre-processing is the method of cleaning the data, choosing the variable to use, and translating the data into a useable format. In this study, the captured data from the surrounding environments are in geojson format. The data will be converted into csv format for each class, then all classes will be combined into one $c s v$ file to be analyzed in the next step.

Next, the prepared data will be analyzed. The goal of this step is to create a model of machine learning to examine the data using different computational techniques and to review the outcome. This begins with the determination of the problem form, the collection of machine learning techniques, the classification of this project, and then the development of the model for evaluation using different machine learning algorithms.

The next step will be training the model. The prepared dataset is used to simulate and train the classification model using Scikit-learn, a library written in the Python language, at a rate of 67\%. Trained data from SVM, KNN, Bagging, and RF classifiers were obtained from Scikit-learn. A model has to be conditioned so that it can understand the different patterns, rules, and features for a better problem outcome. Once a given dataset has been trained on the machine learning algorithm, it will be validated with a new $33 \%$ dataset rate. The model evaluation assesses the model's accuracy according to issue criteria.

\subsection{Comparison with WHO standards}

The prepared dataset is also compared against World Health Organization (WHO) recommendations to check whether it is within the acceptable levels or exceeds the recommended threshold which can cause adverse health effects. In this paper, a graphical user interface (GUI) written in python is used to provide the comparative analysis. Which measures sources of environmental noise from roads, trains, birds and lawn mowers at WHO recommended levels.

\section{RESULTS AND DISCUSSION}

In this section, the performances of SVM, KNN, Bagging, and RF are investigated on four different classes of environmental sounds: highway, railway, lawnmowers, and birds. We use a dataset of 44 samples of environmental sounds that are recorded using a mobile application on a smartphone. Models are trained and tested on a dataset of $67 \%$ and $33 \%$, respectively. We have implemented all algorithms in Python using open-source packages for machine learning that is scikit-learn. The performance of all models is presented with respect to classification accuracy, confusion matrices, and time that is needed for training and testing.

\subsection{Performance evaluation}

To examine the performance of the studied models, the confusion matrix is plotted. The confusion matrix helps in assessing the performance of the model by the source type. Findings are read along the diagonal of the matrix which represents the proportion of agreement between the model and true classified sources. Other results represent the proportion of misclassified data and the source type to which it was incorrectly attributed.

- K-nearest neighbour $(\mathrm{KNN})$ : noise of birds is the class with the lowest misclassification as there is only 1 sample that is misclassified as the noise of highway. The noise of highway is misclassified in 11 samples as noise of train and 4 samples as noise of lawnmowers. The noise of train is misclassified in 13 samples as noise of highway and 1 sample as noise of lawnmowers. Finally, noise of lawnmowers is misclassified in 3 samples as noise of birds and 3 samples as noise of highway as shown in Figure 5(a).

- Support vector machine (SVM): in the previous model, KNN shows 14 misclassification samples for the noise of train class. However, with SVM, there are 18 misclassified samples. On the other hand, the number of misclassified samples decreases from 15 to 1 sample for noise of highway. For noise of train, there are 17 samples misclassified as noise of highway and 1 sample as noise of lawnmowers. For noise of lawnmowers, there are 2 misclassified samples, each for noise of highway and noise of birds. For noise of birds, there is only 1 misclassified sample which is classified as noise of highway as shown in Figure 5(b).

- Bootstrap aggregation (Bagging): regarding the confusion matrix shown in Figure 5(c), Bagging has a similar performance to SVM except for noise of lawnmowers, which has 3 misclassified samples as noise of birds and another 2 samples as noise of highway.

- Random forest (RF): Figure 5(d) shows that RF gives the lowest number of errors compared to other models. There are only two misclassified samples for noise of train: one misclassification for noise of highway and another one for noise of lawnmowers. There is zero error in other classes. RF can be considered the best model for machine-learning based noise classifiers. 


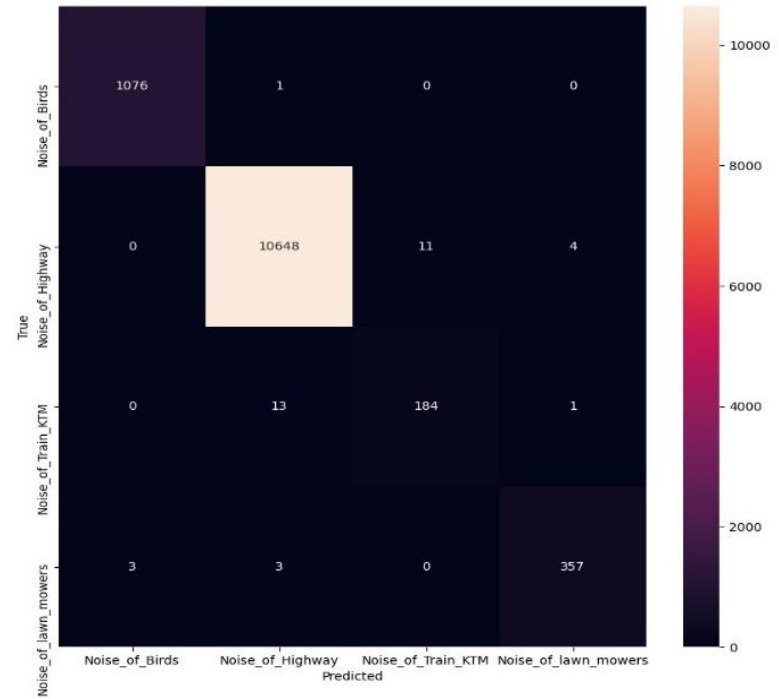

(a)

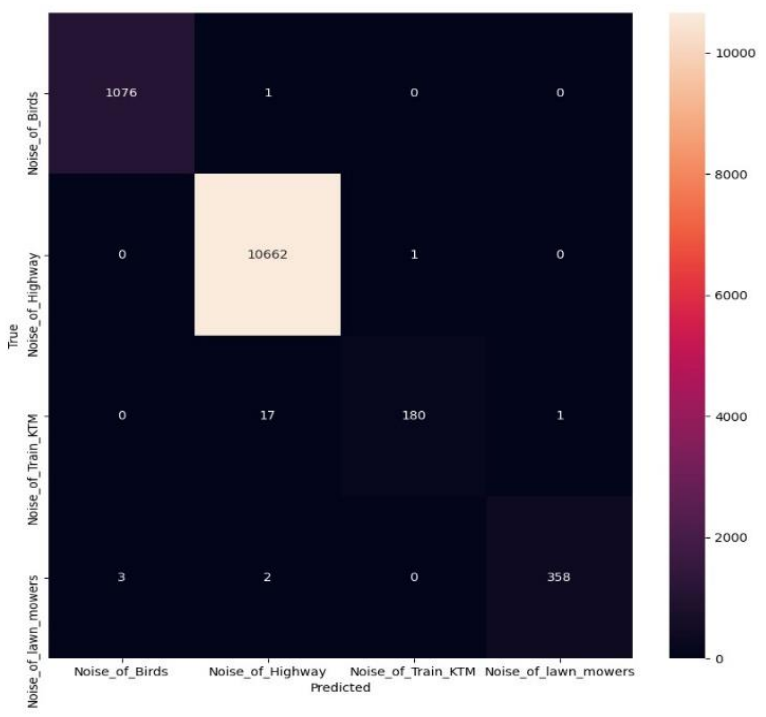

(c)

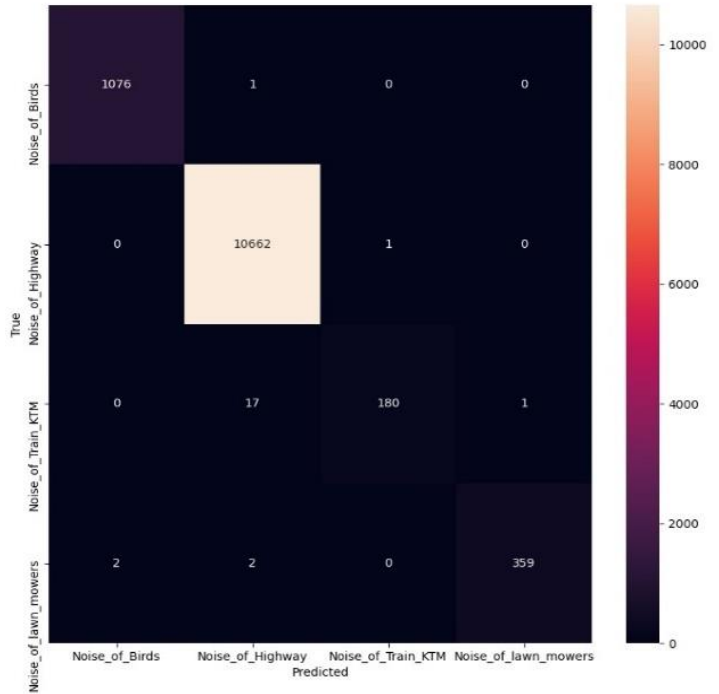

(b)

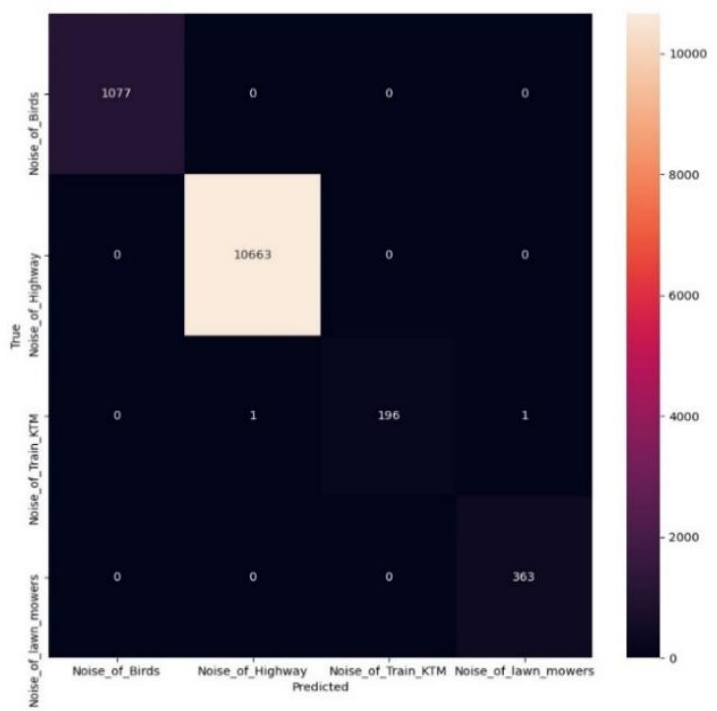

(d)

Figure 5. Confusion matrices of: (a) KNN, (b) SVM, (c) Bagging, and (d) RF

\subsection{Performance comparison of classifiers}

This section discusses the detailed findings of the final classification schemes in terms of accuracy, precision, recall, F-measure, and execution time. In the experiments, the algorithms are compared in terms of the time required to create the individual models, to assess their potential usage in real-time applications. Precision and recall are the basic metrics used to assess the classifier's output quality. In the context of a classification task, precision is the ratio of correctly classified samples to the total number of samples labeled as belonging to this class, whereas recall is the ratio of correctly classified samples to the total number of samples in the database that belong to this class. High scores for both metrics indicate that the classifier produces accurate results (high precision) as well as the majority of all positive outcomes (high recall) [23]. Table 2 lists the performance results of the four studied classifiers.

In terms of noise classification accuracy, all four classifiers perform admirably; the obtained accuracy ranges from $95.3 \%$ to $100 \%$. We may observe that the accuracy of Bagging and RF is slightly higher than the accuracy of KNN and SVM which is contrary to a recent study [19]. Regarding the model training time, the fastest classifier is KNN which corresponds to the cited study [19]. KNN is a type of lazy learning approach that uses all the training data to obtain a prediction. The KNN model produces a great result, however, each time prediction is required, it takes longer than the testing time. As a result, if this model is used in a real-world application, it will be slow. In terms of testing time, RF is the fastest classifier 
while KNN is the slowest. However, in earlier research [19] SVM was the slowest classifier. RF has the lowest prediction time and the highest accuracy, making it the best model since it provides a low prediction time and high accuracy. It also achieves perfect scores for recall, precision, and F1-score. As a result, the model classifies correctly all positive samples and for each class.

Table 2. Performance comparison of classifiers in terms of accuracy, precision, recall, F1 score, and execution time

\begin{tabular}{lcccc}
\hline \multicolumn{1}{c}{ Classifier } & KNN & SVM & Bagging & RF \\
\hline Accuracy [\%] & 95.3 & 96.6 & 98.7 & 100 \\
Precision & 0.93 & 0.94 & 0.96 & 1.0 \\
Recall & 0.97 & 0.97 & 0.97 & 1.0 \\
F1 score & 0.97 & 0.99 & 0.98 & 1.0 \\
Training [s] & 1.01 & 1.91 & 10.56 & 8.36 \\
Testing [s] & 12.9 & 0.68 & 4.75 & 0.12 \\
\hline
\end{tabular}

\subsection{GUI design and implementation}

In this section, the collected noise levels using a smartphone which originated from four different sources that are highway, railway, birds, and lawnmowers, are compared with WHO standards to check whether it is within the recommended levels for health and safety. The comparison is done via a graphical user interface (GUI) written in Python, which is a system of interactive visual components for computer software, displays objects that convey information, and represents actions that can be taken by the user. The main window of the GUI consists of four buttons for four different sources and a text field that is used to display the results of each source based on collected data. After converting the data from JSON format into excel format, it will be read by pandas' library, a software library for data manipulation and analysis. Then, the average value for each source is obtained by adding all values and dividing it with the total length of rows to compare it with WHO standards in the next step.

The WHO standards for highway noise, train noise, birds noise, and mechanical machines noise (lawnmowers) are $53 \mathrm{~dB}, 54 \mathrm{~dB}, 55 \mathrm{~dB}$, and $70 \mathrm{~dB}$, respectively [24], [25]. Once each button is clicked, the result is displayed in the text field as shown in Figure 6(a) for highway, Figure 6(b) for train, Figure 6(c) for birds, and Figure 6(d) for mechanical machines. Results of the comparative analysis with the WHO standards will appear in the text box on the right side of the GUI. The results can be used as a means for automatically screening extraneous noise contributions from environmental monitoring data. In addition, the findings are expected to provide a guideline of the conducive environment for carrying out tasks in the presence of noise.

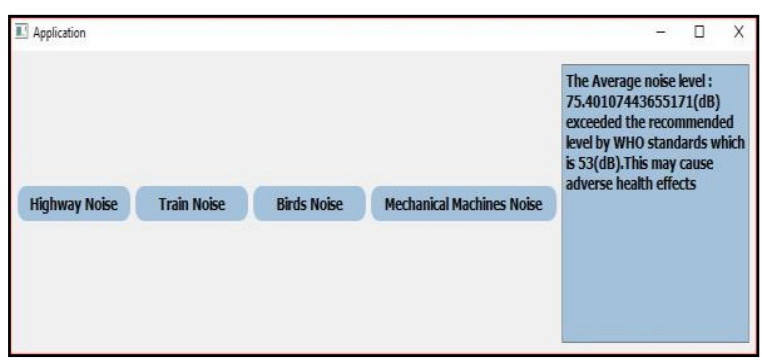

(a)

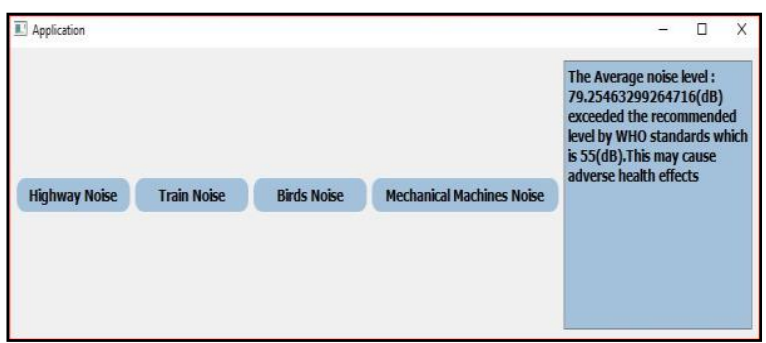

(c)

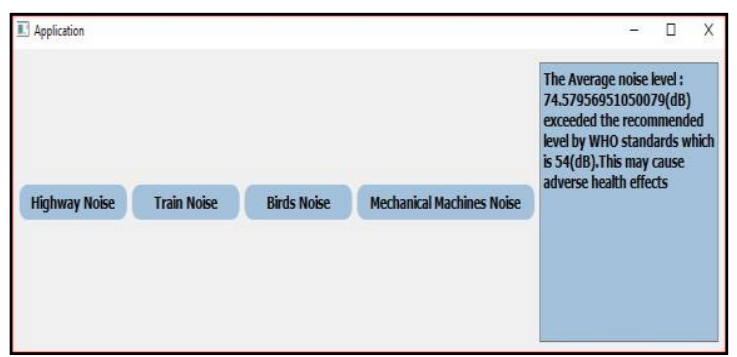

(b)

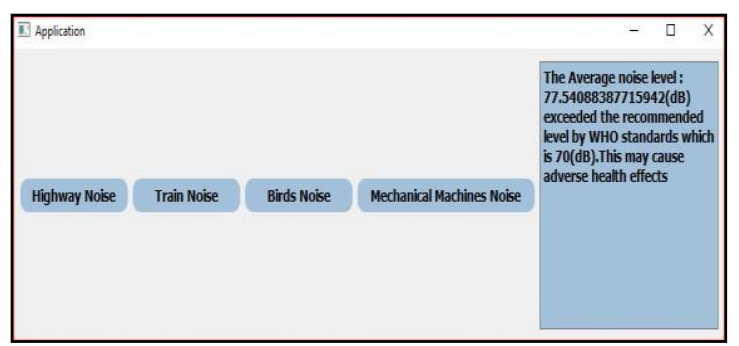

(d)

Figure 6. Windows of each source: (a) highway noise button, (b) train noise button, (c) birds noise button, and (d) mechanical machine noise button 


\section{CONCLUSIONS}

In this paper, we have presented a machine learning approach for noise classification in smart cities. Four supervised machine learning algorithms showed promising performance in classifying four different sound classes: highway, railway, lawnmowers, and birds. It is observed in the study that all models provide high noise classification accuracy that is in the range of $95.3 \%-100 \%$ for various environmental sounds. Regarding the classifiers, RF provided the highest classification rates among the algorithms used in this study. However, although the four algorithms showed good performance, KNN proved significantly faster in constructing the training model, followed by SVM, with RF and Bagging being the slowest. With respect to the testing time, the fastest classifier is the RF and the slowest is KNN. Furthermore, all the captured data from the four different sources of environmental noise in the city of Kuala Lumpur exceeded the WHO recommended levels, which raises the probability of adverse health threats. The findings indicate that machine learning techniques may be utilized effectively in the training of classification models, and also improvements can be obtained by enhancing the methods used to train the model. This is intended to result in the development of more robust models from which to construct a relevant environmental soundscape taxonomy.

\section{ACKNOWLEDGEMENT}

The authors wish to express their gratitude to Ministry of Higher Education (MOHE) Malaysia and Universiti Teknologi Malaysia for the financial support of this project under PY/2021/02061 fundamental research grant scheme titled a machine learning for environmental noise classification in smart cities (Vot number R.J130000.7851.5F428).

\section{REFERENCES}

[1] A. Almehmadi, "Smart city architecture for noise pollution mitigation through the internet of things," International Journal of Computer Science and Network Security, vol. 18, no. 7, pp. 128-133, 2018.

[2] S. A. Stansfeld and M. P. Matheson, "Noise pollution: non-auditory effects on health," British medical bulletin., vol. 68, no. 1, pp. 243-257, Dec. 2003, doi: 10.1093/bmb/ldg033.

[3] T. Kawada, "The Effect of Noise on the Health of Children," Journal of Nippon Medical School, vol. 71, no. 1, pp. 5-10, 2004, doi: $10.1272 /$ jnms.71.5

[4] R. Navarrete et al., "Software and Hardware to Replace a Sound Level Meter," International Journal of Computer and Communication Engineering, vol. 1, no. 4, pp. 297-300, 2012, doi: 10.7763/ijcce.2012.v1.77.

[5] V. Pareek and R. K. Sharma, "Coronary heart disease detection from voice analysis," In 2016 IEEE Students' Conference on Electrical, Electronics and Computer Science (SCEECS), 2016, pp. 1-6, doi: 10.1109/SCEECS.2016.7509344.

[6] Q. Kong, Y. W. Kwony, L. Schreierz, S. Allen, R. Allen, and J. Strauss, "Smartphone-based networks for earthquake detection," 15th International Conference on Innovations for Community Services (I4CS), 2015, pp. 1-8, doi: 10.1109/I4CS.2015.7294490.

[7] J. Daneault, B. Carignan, C. É. Codère, A. F. Sadikot, and C. Duval, "Using a smart phone as a standalone platform for detection and monitoring of pathological tremors," Frontiers in human neuroscience, 2012, doi: 10.3389/fnhum.2012.00357.

[8] G. Ma, Y. Tian, T. Ju, and Z. Ren, "Assessment of Traffic Noise Pollution from 1989 to 2003 in Lanzhou City," Environmental monitoring and assessment, vol. 123, no. 1, pp. 413-430, 2006, doi: 10.1007/s10661-006-1494-6.

[9] L. A. Rahim, M. Hashim, and N. Nayan, "Road Traffic Noise Pollution and its Management in Tanjong Malim, Perak," Journal of Techno Social, vol. 3, no. 2, 2011

[10] M. Mehdi, M. Kim, J. Seong, and M. Arsalan, "Spatio-temporal pattern of road traffic noise pollution in Karachi, Pakistan," Environment international, vol. 37, no. 1, pp. 97-104, 2011, doi: 10.1016/j.envint.2010.08.003.

[11] Z. Hj. Ibrahim and H. K. Richard, "Noise Pollution at School Environment Located in Residential Area," Malaysian Journal of Civil Engineering, vol. 12, no. 2, 2018, doi: 10.11113/mjce.v12.15636.

[12] R. E. Hayden, "Roadside noise from the interaction of a rolling tire with the road surface," The Journal of the Acoustical Society of America, vol. 50, no. 1A, p. 113, 2005, doi: 10.1121/1.1977497.

[13] D. Ouis, “Annoyance From Road Traffic Noise: A Review," Journal of environmental psychology, vol. 21, no. 1, pp. 101-120, 2001, doi: 10.1006/jevp.2000.0187.

[14] U. N. D. E. S. Affairs, "World Urbanization Prospects: The 2018 Revision," in UN-iLibrary, New York, United States, doi: 10.18356/b9e995fe-en. [Online]. Available: https://www.un-ilibrary.org/content/books/9789210043144.

[15] H. Chourabi et al., "Understanding Smart Cities: An Integrative Framework," in 2012 45th Hawaii International Conference on System Sciences, 2012, pp. 2289-2297, doi: 10.1109/HICSS.2012.615.

[16] R. Giffinger, G. Haindlmaier, H. Kramar, and F. Strohmayer, "European smart cities 4.0," 2015.

[17] C. M. Bishop, "Pattern Recognition and Machine Learning," Springer Innovations in Communication and Computing, 2006, doi: 10.1007/978-3-030-57077-4_11.

[18] J. Picaut, N. Fortin, E. Bocher, G. Petit, P. Aumond, and G. Guillaume, "An open-science crowdsourcing approach for producing community noise maps using smartphones," Building and Environment, vol. 148, 2019, doi: 10.1016/j.buildenv.2018.10.049.

[19] Y. Alsouda, S. Pllana, and A. Kurti, "IoT-based urban noise identification using machine learning: performance of SVM, KNN, Bagging, and Random Forest," Proceedings of the international conference on omni-layer intelligent systems, 2019, pp. 62-67, doi: 10.1145/3312614.3312631.

[20] M. Sahidullah and G. Saha, "Design, analysis and experimental evaluation of block based transformation in MFCC computation for speaker recognition," Speech Communication, vol. 54, no. 4, pp. 543-565, 2012, doi: 10.1016/j.specom.2011.11.004.

[21] F. Pedregosa et al., "Scikit-learn: machine learning in Python," Journal of machine Learning research, vol. 12, no. 85, pp. 2825-2830, 2011. 
[22] E. Bocher, G. Petit, J. Picaut, N. Fortin, and G. Guillaume, "Collaborative noise data collected from smartphones," Data in Brief, vol. 14, pp. 498-503, 2017, doi: 10.1016/j.dib.2017.07.039.

[23] D. Powers, "Evaluation: from precision, recall and f-factor to roc, informedness, markedness \& correlation," Machine Learning Technology, vol. 2, no. 1, Jan. 2008.

[24] B. Berglund, T. Lindvall, D. H. Schwela, W. H. O. O, and E. H. Team, "Guidelines for community noise," World Health Organization, 2000.

[25] Z. Jakab, "Environmental noise guidelines for the european region," WHO Regional Office for Europe, 2018.

\section{BIOGRAPHIES OF AUTHORS}
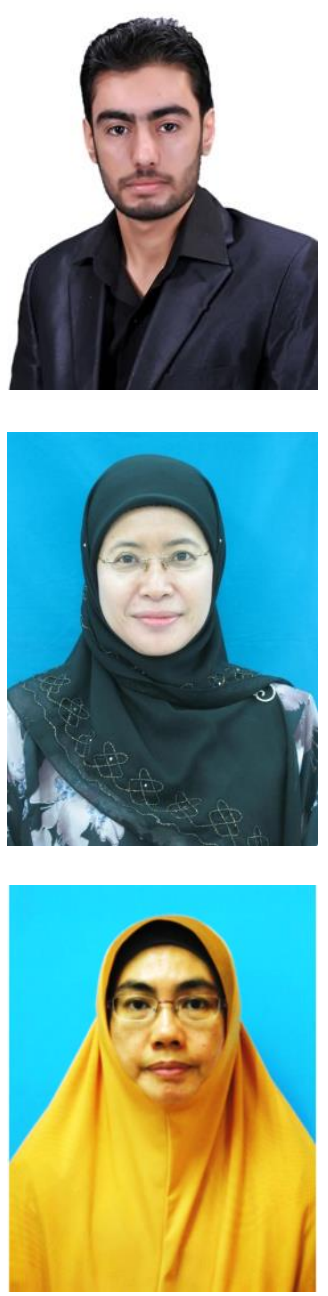

Yaseen Hadi Ali (iD id SC P received the bachelor's degree in electrical engineering from University of Anbar, Anbar, Iraq, in 2017, and the Master's degree in electronics and telecommunication engineering from University Technology Malaysia, Johor Bahru, Malaysia in 2021. He is currently a lecturer in computer techniques department, Alsalam University College, Baghdad, Iraq. His research interests are machine learning (ML), internet-of-things (IoT) and wireless sensor network (WSN). He can be contacted at email: Yaseenhadi5@gmail.com.

Rozeha A. Rashid (iD 8) SC P received her B. Sc. degree in electrical engineering from the University of Michigan, Ann Arbor, USA and her M.E.E. and PhD degrees in telecommunication engineering from Universiti Teknologi Malaysia (UTM). She is an associate professor in the department of communication engineering, school of electrical engineering, Universiti Teknologi Malaysia and is currently the head of telecommunication software and system (TeSS) research group. Her current research interests include wireless communications, sensor network, cognitive radio and internet-of-things. She can be contacted at email: rozeha@utm.my.

Siti Zaleha Abdul Hamid (D) SC P received her B. Sc. degree in computer and communication engineering from Universiti Sains Malaysia (USM) and her M. Sc. in Sound and vibration studies from University of Southampton, England. She is a senior lecturer in the department of communication engineering, school of electrical engineering, Universiti Teknologi Malaysia and is currently a member of telecommunication software and system (TeSS) research group. Her current research interests include sound and acoustic engineering, Internet-of-Things and engineering education. She can be contacted at email: szaleha@utm.my. 